\title{
The Impact of Emerging Technologies on Knowledge Management in Organizations
}

\author{
Tarek A. El Badawy ${ }^{1}$, Rania M. Marwan ${ }^{2} \&$ Mariam M. Magdy ${ }^{1}$ \\ ${ }^{1}$ Faculty of Management Technology, German University in Cairo, Cairo, Egypt \\ ${ }^{2}$ Ain Shams University, Cairo, Egypt \\ Correspondence: Tarek A. El Badawy, Assistant Professor of Management, Department of Management and \\ Organization, Faculty of Management Technology, German University in Cairo, Cairo, Egypt. E-mail: \\ tarek.el-badawy@guc.edu.eg
}

Received: January 27, 2015

Accepted: March 4, 2015

Online Published: April 25, 2015

doi:10.5539/ibr.v8n5p111

URL: http://dx.doi.org/10.5539/ibr.v8n5p111

\begin{abstract}
Man versus the machine, it is a new and growing controversy that is irking those who criticize the increased use of technology, while considered to be extremely exciting news for supporters and advocators of the growing technologies in western countries. Supporters of the rapid technological advancement have argued that it has definitely increased productivity, and improved the economy. On the other hand, the opposing side has argued that such technologies are destroying jobs more than it is generating new ones. This paper examines the impact of growing technologies as depicted by the introduction of Watson the supercomputer, which represents the epitome of technological advancement, on knowledge management in organizations, by focusing on three important components that shape any business; people as identified by managers and employees, organizational processes, and the emerging technologies. The paper proposes a framework for managing knowledge and knowledge workers as well as proposing an adapted prototype design of how the future digitized organizational structure should be, and offers recommendations in this regard.
\end{abstract}

Keywords: knowledge management, emerging technologies, creativity, innovation

\section{Introduction}

Knowledge Management is the interface of people, processes, and technology. It is the sum of what is known (tacit and explicit knowledge), and it resides in the intelligence and the competence of people (Nemati, Steiger, Iyer, \& Herschel, 2002). It is an interdisciplinary concept of exact and inexact sciences (Sharara, 1997). It is software-support handling of things through storing, administering, retrieving of objects used in connection with Information Communication Technology (ICT) in businesses (Maier, 2007). Rapidly changing technologies have changed the future of businesses, consequently the different roles played by the components of knowledge management; people, process, and technology are yet to be defined in this changed future image. Especially the way organizational knowledge is created and managed in these organizations.

As the world is experiencing rapid advancement in technology that is changing the face of business, fewer people are working in manufacturing in the U.S and in China than in the 1997, and this is due to the increased automation of plants. Examples of technology in manufacturing are robots that are used to move around things in warehouses, and Google's driverless car (Rotman, 2013). Other simpler technologies are the Web, artificial intelligence, which have dramatically affected employment, as many white collar employees have been displaced by these technologies. Jobs in the post office and in customer service have disappeared (Rotman, 2013).

The most recent advancement is IBM's Watson supercomputer. Watson is able to rapidly gather and selectively display information tailored to the moment and to the needs of individuals (Taft, 2011). It can understand and process natural language. It can generate and evaluate hypotheses to provide valid responses with a confidence level. It has a dynamic learning capability, it can be trained. It communicates more like a human. It can process 500 gigabytes (1million books per second) (Abeles, 2012). It is able to deal with ambiguity; it displays human traits, and is able to resolve complex problems quickly (Petro, 2012).

The purpose of this paper is to explore the literature on technology and its effect on knowledge management (KM), KM components and the organization's transition into becoming a learning and knowledge creating entity. 
This drove the researchers to pose the following research questions: $\mathbf{R Q}_{\mathbf{1}}$ : To what extent will advanced technology, as depicted by Watson the supercomputer, affect the knowledge management components of the organization, specifically the people's component? $\mathbf{R Q}_{2}$ : How will the future organization look like?

The importance of the paper stems from the fact that organizations seek efficiency and effectiveness in their operations. Emerging technologies, if correctly utilized, will help the organization seek its goals. Technology allows employees and employers make the best decisions and utilize their resources in order to become sustainable and reach a competitive edge. The proposed framework for the digitized organization helps decision makers in understanding the aspects of change. Consequently, they will be able to correctly plan and execute a transition strategy.

The paper starts with the literature review on technology and its relation to employees in terms of the challenges it imposes on the individual's skills and job requirements. This is followed by discussing the knowledge creating process in the organization. Finally, the authors present their opinion on the outlook of the future digitized organization as depicted by integrating Watson the supercomputer.

\section{Technology and Knowledge Management}

Gates (1999) argues that knowledge workers are the brains of the company; they cannot be disconnected from the company's important data. He believes that if the right information is available for workers and accessible in a matter of minutes and not days, and if the right people are working on this accessible data, then the company will gain huge advantage. He further believes that the digital information flow is not only giving power to employees in achieving and maintaining competitive advantage, but also is a revolutionary change in the structure of processes, that is more important than any other change since the introduction of mass production (1999).

Gates addresses the impact of digitization on the knowledge management components of the organization; that is technology as represented by the digital age; the right people working on it will change the structure of the work processes for the best. Gates is one of the major supporters of the introduction of technology into businesses; he believes that the industrial age has extended the capacities of muscles, whereas the digital age extended the capacities of minds. He asserts that a business that has a digital nervous system can help define itself and its role in the future, however the success and failure depends on the leader (1999).

Other supporters of technology argue that the pressures on employment in the States specifically, are not necessarily due to computers, there are other factors that might have caused such a decline, such as the financial crises in the early and late 2011 (Rotman, 2013). On the other hand, the opposing side of the digitization of organizations acknowledges that without doubt productivity will increase and there will be improvement in the efficiency and effectiveness of the work processes, yet the people component will dramatically be affected. Furthermore, important jobs will definitely disappear, compounded by the exponential advance of digital technologies, the result would be the alienation of people in businesses; they won't be good enough to run the show! (Rotman, 2013).

\section{Technology and People}

Rotman looks back at history; in the 1980s, technology has taken over jobs such as bookkeeping, clerical work, and repetitive production jobs in the manufacturing sector. On the other hand, the demand on higher and lower paying jobs has increased, adding to the "polarization" of the workforce, and the wiping out of the middle class. This pattern has been repeating itself for the last decades in many of the industrialized countries, and it is a pattern that reflects a change in the type of jobs, but not a change in the total number of jobs (2013).

Lawrence Katz, a Harvard economist confirms the notion that technology will only lead to a mismatch between the needs of employers and the skills of workers, he asserts that there is no historical pattern that shows technological advancement leading to a net decrease in jobs over an extended period of time. The only problem that will proliferate is the required time to acquire the necessary skills, but Katz dismisses that by saying that people create new jobs (Rotman, 2013). In other words, technology is widening the gap between those who are tech savvy, and those who are not. John Leonard, a professor of engineering at MIT believes that despite the accelerated digitization, people will still excel at dealing with change and with unexpected events, and that the idea of people and machines working together is more feasible than machines displacing people (Rotman, 2013). It still remains to be a relatively unknown predicament of people in the future digitized organizations, yet what can be assured is that with the advancement in technology, the type of work and the skills necessary to perform this work will definitely change.

The people component in knowledge management can be categorized into managers and employees. According 
to H. Sharara (2014a) there are three types of managers in organizations, there are those who:

1) Manage daily results (short, important, urgent);

2) Develop people (medium range, important);

3) Improve (Long range, important).

The first type of managers spends $50 \%$ of his/her time managing daily problems, $30 \%$ developing employees, and $20 \%$ on innovation. He is just a muscle that is working more than the required amount of hours, and pushing employees (Sharara, 2014a). In the new digitized organization, this type of managers is obsolete and doomed for failure.

The second type develops employees according to SOPs. The procedure is definitely leaner and more efficient, employees need less attention and time is saved. Promotion is based on managing daily activities (Sharara, 2014a). In the new digitized organization, this type of managers is also obsolete, since many of the redundant tasks that he would be performing would be hijacked by technology or Watson the supercomputer.

The first two types of managers are myopic in two important aspects; the first one in not realizing that the nature of many things in business is that they complement each other and that management is all about the intersection of things (Sharara, 1997). The new digitized organization calls for the combined efforts of both vertical and lateral thinking, which represents the essence for innovation (DeBono, 1990). The other important aspect is that they are myopic by focusing on survival, while ignoring adaptation, evolution and growth, and this dooms the organization for failure in a highly digitized competitive market (Sharara, 1997).

The third type is a leader who takes risks, while being rational. In this case the pyramid is inverted; he spends $50 \%$ of the time improving his subordinates, $30 \%$ developing, and $20 \%$ managing by objectives (MBO) (Sharara, 2014a). His role is to evaluate the whole environment of the work, utilizing his para knowledge; philosophizing, fantasizing and materializing. He is making use of his lateral knowledge, and educating his employees on how to switch from vertical sequential way of thinking, as the above two managers, and to focus more on looking at things in a different way, which is known as the lateral thinking (Adair, 2007). Watson is there to perform the traditional rudimentary way of thinking, which the leader and his team definitely take, and restructure it, provoke it, and dig for as many alternatives around it. The leader and his team add creativity to the vertical thinking supplied by Watson, and develops lateral thinking as an attitude of mind and a process within the organization.

This leader is concerned with concept breaking, provocation, and disruption of given ideas to allow for the restructuring of patterns (DeBono, 1990), he is concerned with developing creative chaos (Schon, 1983). Watson will cover the inductive and deductive logic, while the leader will be concerned with the abduction part, which is the lateral extension of the reasoning process (Nonaka, 1994).

The second categorization of people is employees. As was mentioned before, the type and skills needed in the new digitized organization will differ. Acquiring new skills will be an important factor for success, and a cornerstone in the comprehensive evolution of management towards the new digitized form. With the introduction of Watson the supercomputer, as the current epitome of technological advancement, and the shrinking of the size of employees versus the machines, the new digitized organization will rely on a selected number of employees, who will act as the "guiding nucleus" of the organization towards its evolution. This guiding nucleus will contribute to the evolution of the organization by being mentally fit (Sharara, 1997, p. 29), and by revising the thinking process in the organizational knowledge creation process (Nonaka, 1994).

\section{Employee Mental Fitness}

Mental fitness for employees is making sure that they are enjoying a balanced life socially, physically, mentally, and spiritually. In neuroscience, it is increasing organized networks among neurons, which is the result of willful, purposeful, and active learning. Mental fitness is a precursor to lateral thinking and creativity. Total mental fitness is the proactive balance among the interaction of analytical thinking, social communication and creative thinking. The goal is to make sure that the selected guided nucleus of employees are able to extend their reasoning laterally, and think in a different way, and instill this as a process in the new digitized organization. This will not take place unless they are mentally fit to do so.

Google's efforts to make its employees mentally fit, includes providing international cuisines for all nationalities, other companies offer a place for employees to nap, and some provide a place for employees to do their laundry. Other organizations provide an environment that is conducive for creativity like having an aquarium within its premises. All are efforts to tap into the creative mind of employees, to provoke the lateral process of thinking. 


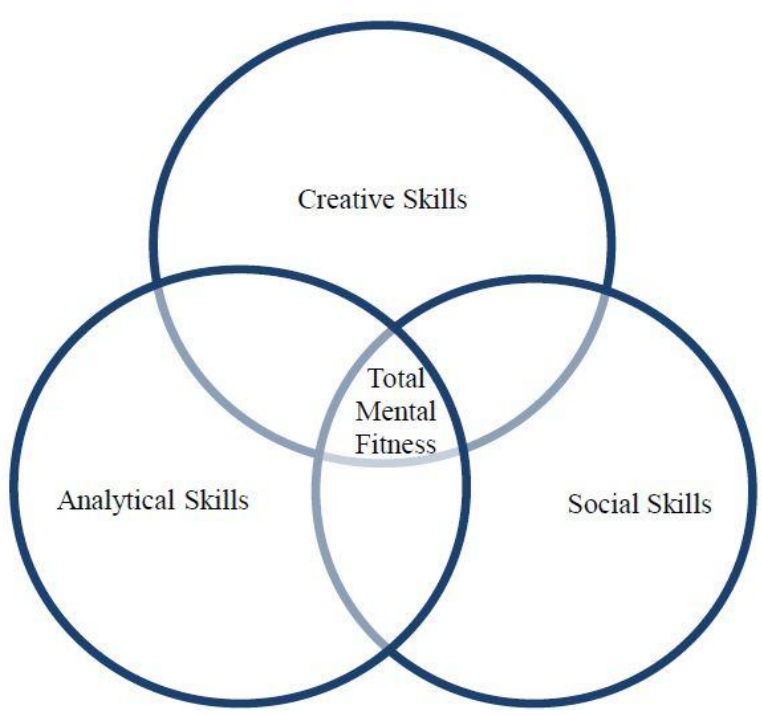

Figure 1. Total mental fitness

The lateral process of thinking refers to a 360-degree thinking. In other words, it is generating as many approaches as possible, as opposed to selecting only one best approach (vertical thinking). The 360-degree thinking will lead to a total balanced mental fitness, that is based on the intersection (the AND) of the analytical thinking, social thinking, and creative thinking (not EITHER /OR) as depicted in Figure 1. In other words, it resembles not focusing all efforts on one closed angle (Sharara, 1997).

Instilling a 360-degree thinking system requires the guiding nucleus of employees to acquire certain habits so as to break the mind free from the usual patterns, and train it to restructure preconceived regular routes of thinking. In a lecture to a Knowledge Management and Innovation class on December 12, 2014, H. Sharara discussed that developing 7.5 habits would equip the guiding nucleus of employees to be mentally fit for 360-degrees process of thinking. He explained how the 7.5 habits would open the door to the lateral process of thinking. The following is a brief description of the 7.5 habits (2014b).

Habit 1 refers to jumping out of the box, for lateral thinking calls for taking leaps, and not moving in sequential steps as in vertical thinking. De Bono says "it may be necessary to be on top of the mountain to find the best way up" (1990). This is done by constantly challenging assumptions, whether they are unconscious or established or professional solutions. In lateral thinking nothing is lost, information is tackled in a provocative way to achieve re-patterning, which is what is required in the new digitized organization. This involves tapping into the unconscious, and deliberately seeking irrelevant information, putting assumptions and challenging them, and deploying the most promising. In this way the span of relevance is widened, and 360-degree knowledge is deployed through a 360-degree lateral thinking.

Thinking out of the box includes defining the problem correctly when faced by it, which is a solution in disguise. There are three types of problems according to De Bono, the first one entails information gathering, and improving the technique. This represents a vertical way of thinking that would be made extremely accessible and easy by the introduction of Watson into the organizations. The second type entails rearrangement of information, insight restructuring, provoking disrupting and breaking concepts. The third type is the problem of being stuck in the current situation, unable to move to a better one (De Bono, 1990). The second and the third types are approached through a 360-degree of lateral thinking, hence not digging the hole deeper, but rather digging the hole in a different place.

Habit 2 calls for the invitation of chance intrusions, for many major inventions were made as a result of chance. Many inventions that we use in our daily life were the result of strange beginnings, like the roll-on deodorant and the microwave oven. The roll-on deodorant was invented in 1988 in Philadelphia, and the inspiration for it was the ballpoint pen. The microwave oven was invented as a by-product of World War 2 radar research, the engineer Percy Le Baron Spencer was working on magnetrons when the bar of chocolate in his pocket melted. By chance he discovered it, and began to further experiment and discovered that microwaves could cook food in a much shorter time than normal (Bailey \& Black, 2012). Serendipity has to be followed by a proactive attitude towards 
chance events, otherwise it will just pass undiscovered, and thus it falls upon individuals to manage their luck carefully by being purposeful, perceptive and sensitive to everything around, or as Louis Pasteur said "chance only favors the prepared mind". Lateral thinking is playing around with experiments, notations, ideas and models; it provokes a 360-degree way of thinking that encompasses the relevant and the irrelevant.

Habit 3 calls for the listening to the moaning of your depth mind, the depth mind makes a range of combinations of the meta- process of analyzing, criticizing and synthesizing. It is considered to be the womb of thinking, then the creative process comes to practice by linking the conscious mind with the unconscious mind. Soichiro Honda was an engineer, a creative thinker and an innovator. When he built his first motorcycle, he was not very much impressed with the way it looked, as it was made by his designers. He thought it looked ugly, so he took a break, and while he was sitting in one of the temples, probably meditating, he was fascinated by Buddha's face, and then he made the meta-processes combinations, he imagined a resemblance between Buddha and his motorcycle. He gathered the necessary information by studying Buddha's face in other temples. He worked with his designers to come up with a smooth looking motorcycle that reflected the beauty he had seen in Buddha (Adair, 2007). Soichiro went from preparation (building a motorcycle without a clear vision of how it would look, and delegating this to his designers, who did not do a very good job) to incubation (taking a break and sleeping over it) to illumination (sitting in the temple when least expected and idea struck! Why not make the motorcycle look like Buddha?) to verification (having an action plan that would reflect the beauty he saw in Buddha on the design of his motorcycle.

Habit 4 calls for postponing judgment. The purpose for that is to give room for the idea to breathe, and maybe possibilities of new ideas to be revealed. Avoid idea killers who are narrow minded and egocentric. Avoid colliding communication, and seek out constructive communication through lateral thinking and parallel communication through six thinking hats.

Habit 5 calls for using analogies extensively. Creative thinking is thinking analogically, rather than thinking logically. Using metaphors provides a comprehensive view of things, like perceiving organizations as mechanistic and organismic systems. Mechanistic systems are like machines, when put into operation will go on and on serving its predetermined purpose. Organismic systems are like living organisms, which survive, adapt, evolve and reproduce. Through these metaphors one understands that for the comprehensive evolution of management, organizations need to be viewed at as mechano-organismic systems; mechanistic for survival, the "here" and "now" concern of managers, and organismic for adaptation, and growth, the often neglected "there" and "tomorrow" (Sharara, 1997).

Habit 6 calls for welcoming ambiguity. The common funnel of decision making. This should be done with courage, perseverance, preparation, delegation to the depth mind, and recreation. For creativity to be acquired and systemized, it needs to be disciplined. In other words, discipline yourself to creativity; do not wait for the apple to fall off the tree. Naguib Mahfouz, the Egyptian Nobel Laureate in literature disciplined himself to sit and write every afternoon after he returned from work; he did that all his life whether he found something to write or not.

Habit 7 calls for storing your ideas, for the thinking process requires stored knowledge as inputs.

7sub habits of the $7^{\text {th }}$ habit curiosity to learn reflects a fit mind. Careful and analytical observation at the meta-process of your mind puts the mind in shape for lateral reasoning. Master the art of listening to what is being said and what is not, and postpone judgment. Lateral knowledge is knowledge in every field, the best way to achieve this is through reading. Traveling provides exposure to other habits and other cultures, digging different holes to explore lateral versus vertical reasoning. Playing games requires different skills; physical, mental, and social skills and it depicts a fit mind. Record ideas as they tend to fly away like feathers, you cannot find them, capture the idea and record it.

\section{Technology and the Organizational Knowledge-Creation Process}

People are the creators of knowledge within the organization. Knowledge is created through conversion between tacit and explicit knowledge, generating four different modes of knowledge conversion (Hall, 2005; Nonaka, 1991).

1) Tacit to tacit;

2) Explicit to explicit;

3) Tacit to explicit;

4) Explicit to tacit. 
Organizational knowledge-creation can be viewed as an upward spiral process, starting at the individual level moving up to the collective level, and then the organizational level. Sometimes it reaches the inter-organizational level (Nonaka, 1997).

It is important to note that Watson in the digitized organization will become a cornerstone in the creation of knowledge, being able to understand natural language and to communicate more like a human, it will definitely contribute to the model shift and spiral knowledge.

The spiral model is formed by the dynamic interaction of the four modes of conversion, to form a continual cycle. The cycle is shaped by a series of shifts between the different modes of knowledge conversion (Bolloju, Khalifa, \& Turban, 2002). Watson's role will be more like a catalyst in the chemical reaction, increasing the speed, and enlarging the scale of the spiral conversion by making all sorts of information available at a glance, which will in turn positively affect the way work is processed, and consequently the competitiveness of the organization. The question that remains is that whether or not there will be room for organizations that are not technologically savvy, as the gap between those who are and those who are not will definitely get wider with time.

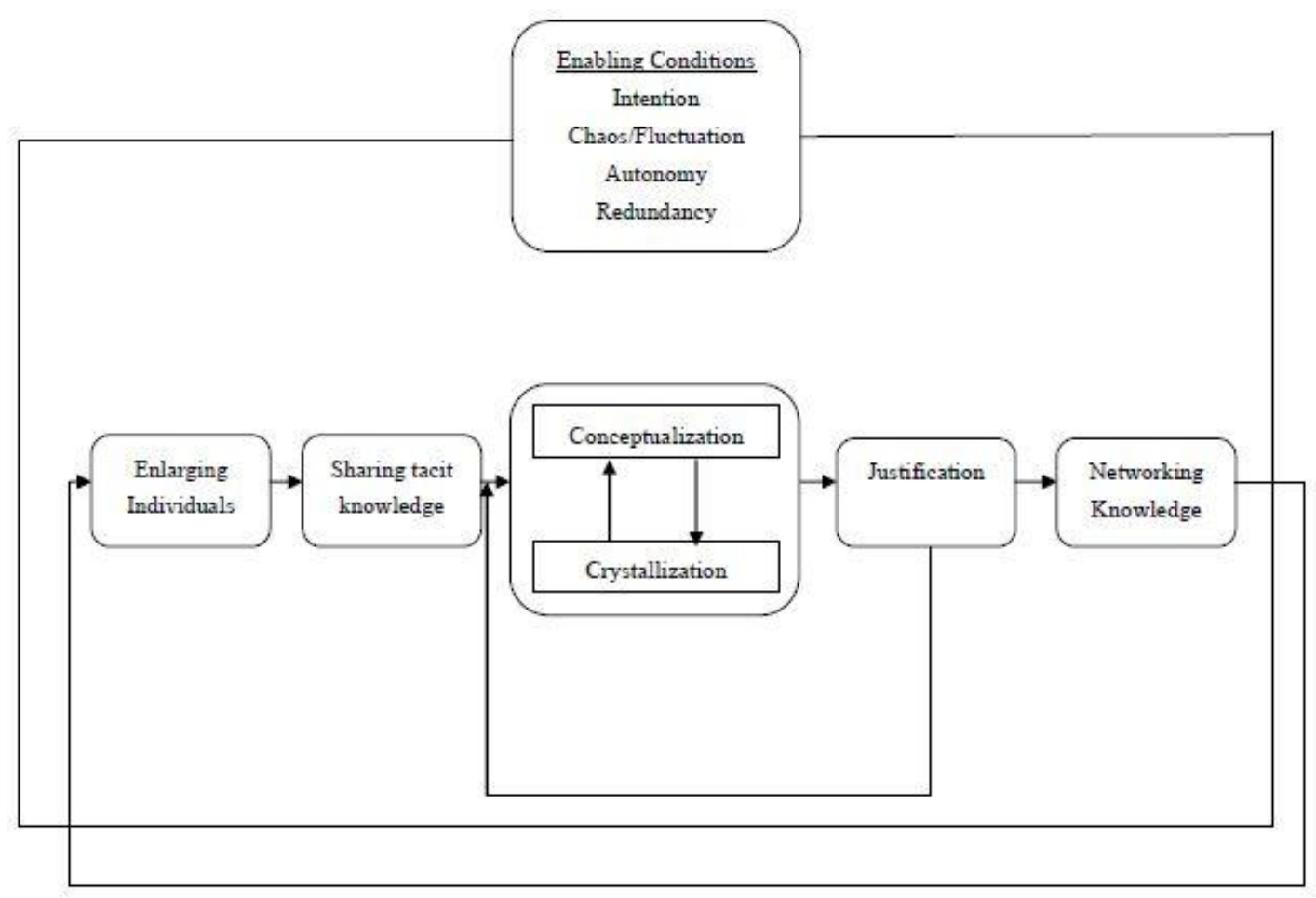

Figure 2. Organizational knowledge-creation process

Figure 2 shows the process of organizational knowledge creation (Nonaka, 1994). In the knowledge-creation process, knowledge is induced through shifts, from socialization and the building of teams and force fields, to the sharing of experiences, then successive dialogue which reveals hidden tacit knowledge (externalization). This is the spiral model, which enlarges the process of knowledge-creation, added to this process the exponential abilities of Watson in making combinations in the shortest of times, then the results are definitely exciting, and would definitely have positive repercussions on the organization (Nonaka, 1994).

The interaction rhythms of teams to create knowledge goes through a process of divergence and convergence (spiral amplification), and is balanced by the leader to converge into a concept, which is then crystallized, justified and communicated forming a network knowledge. The process of creation of a concept is done by three processes: Induction, deduction and abduction (Nonaka, 1994). The first two are the vertical way of reasoning; which, with the help of Watson in the digitized organization, would be extremely facilitated. On the other hand, the abduction part refers to the lateral extension of reasoning, the 360-degree thinking that will be considered an important skill that needs to be educated and applied in the new digitized organization. 


\section{The Future Organization}

Following the literature reviewed, the authors believe that, with the introduction of Watson the supercomputer, the leader/manager will spend more than 50\% of his/her time not just improving employees, but provoking, disrupting and shaking preconceived ideas to bring out the best within the subordinates. As Sharara (1997) explains, to think the impossible and relate the inconceivable.

In the new digitized organization, machines will definitely displace employees, advocators and the opposing parties of the technologies agree that new skills will need to be mastered by the employees in order to fit in the new setting. A few selected employees will be hired in this digitized organization as the "guiding nucleus" towards its success. These selected few will need to be mentally fit in order to practice the 360-degree of thinking that is the wild lateral thinking process that transcends the human experience. They can become mentally fit by following the 7.5 habits of effective thinking previously highlighted.

The knowledge-creation process together with the advanced Watson technology will cause an exponential enlargement of the scale of the spiral conversion, and increasing the speed by making all sorts of information available at a glance. The question that will still remain is whether this would alienate and marginalize organizations that are not digitized.

Watson the supercomputer which has the capability of manipulating large data sets using a variety of analytical and display tools, will definitely need a different organizational fabric than the usual one to incorporate such changes. Two internal environmental organizational factors will need to be revisited; the thinking unit within the organization and the organizational structure that houses it.

The framework for managing knowledge and knowledge workers is through a new thinking unit that will guide the digitized organization to success, which is the TRIAD (Sharara, 1997). The thinking unit will no longer be the individual, but rather a trio of comprehensive knowledge sources: Creative thinking, lateral thinking and real-time information technology. The new technology will hijack human thinking, analytical thinking and some pseudo-creative thinking, especially with Watson that understands human language, and even picks up potty words.

Knowledge management is not only about the creation of knowledge, and the different tools and support systems that facilitate this process, but also about how the knowledge flow is organized, and communities of practice are established (Le Blanc \& Bouillon, 2012). The proposed organizational structure is a prototype design of a knowledge creating organization adapted from Nonaka (1994) as shown in Figure 3. It is a hypertext organization, which is a concept borrowed from computer science that links concepts and allows for the viewing of problems from different perspectives (Jacques, 1979). It is multilayered, having a knowledge base, business system and a project team. Layer 1 will be Watson's specialty, incorporating tacit and explicit knowledge. Layer 2 is the regular routine operation of the business system. The top layer is self-organizing teams of the "guiding nucleus", which are loosely linked to each other, and share the corporate vision. The idea is that the hypertext organization is play-dough like; it takes different forms according to the perspective from which it is seen. The structure reflects a mechano-organismic system, where information traverses across the three layers in a dynamic living cycle of knowledge. Teams are involved in creating knowledge, fluidly interacting with other project teams. Once a team finishes its task, it moves down to the knowledge base to store the information. They then move to routine operations in the business layer until they are assigned another project.

The ability to shift easily in-between the three layers is key to the success of the organization in the digitized era, since the guiding nucleus of employees are a few selected people, who are multi-skilled, and who are constantly creating knowledge with the aid of the supercomputer, tapping into their lateral thinking. 


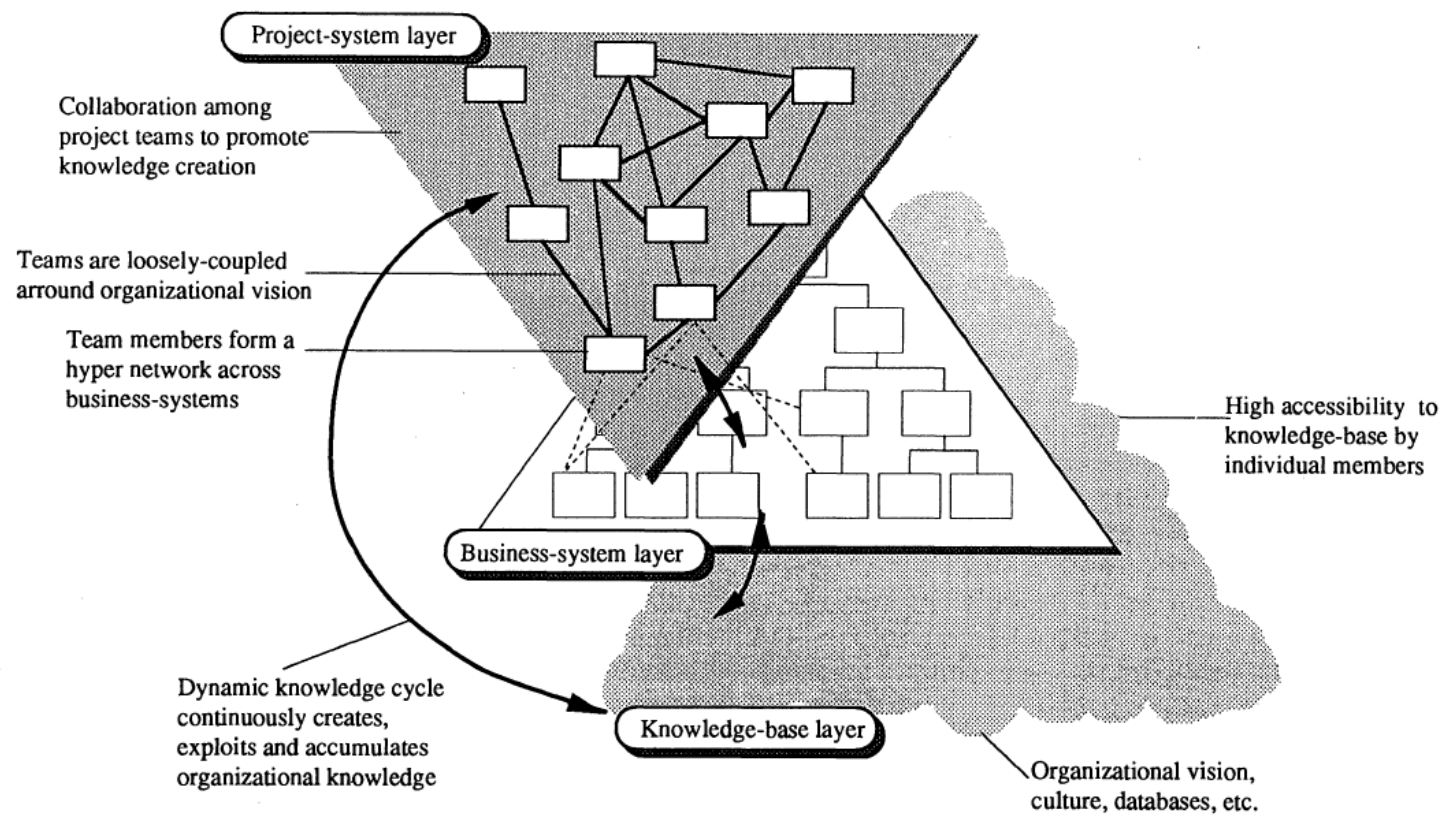

Figure 3. Prototype of the organizational design of the digitized organization

The manager/leader in this organization manages chaos, since creativity and routine do not go together. He/She directs chaos into useful knowledge, by giving teams a conceptual framework to work with, and that which is aligned with their experience. The organization is on a continuous mission to evolve, and reproduce synergistic knowledge with the help of cutting edge technology, where the combined effect is greater than the sum of the parts.

In conclusion, emerging technologies are transcending in organizations and imposing challenges in the management and business field. The new technology requires change in organizational culture, nature of jobs, skills and capabilities and others. Decision makers and strategic management practitioners must pay attention to the future digitized organization if they seek sustainability and creation a competitive edge.

\section{References}

Abeles, T. (2012). After Watson. On the Horizon, 20(1), 3-6. http://dx.doi.org/10.1108/10748121211202017

Adair, J. (2007). The art of creative thinking: How to be innovative and develop great ideas. London: Kogan Page Limited.

Bailey, S., \& Black, O. (2012). The mind gym. London: Little Brown Book Group.

Bolloju, N., Khalifa, M., \& Turban, E. (2002). Integrating knowledge management into enterprise environments for the next generation decision support. Decision Support Systems, 33, 163-176. http://dx.doi.org/10.1016/S0167-9236(01)00142-7

De Bono, E. (1990). Lateral thinking: A textbook of creativity. London: Penguin Books.

Gates, B. (1999). Business @ the speed of thought. London: Penguin Books.

Hall, H. (2005). Knowledge management in times of change: Tacit and explicit knowledge transfers. Doctoral Dissertation, University Of North Texas, Texas, USA.

Jacques, E. (1979). Taking time seriously in evaluating jobs. Harvard Business Review, September-October, 124-132.

Le Blanc, B., \& Bouillon, J. (2012). Organizational devices for knowledge management. VINE, 42(3/4), 382-395. http://dx.doi.org/10.1108/03055721211267503

Maier, R. (2007). Knowledge management systems. New York: Springer.

Nemati, H., Steiger, D., Iyer, L., \& Herschel, L. (2002). Knowledge warehouse: An architectural integration of knowledge management, decision support, artificial intelligence and data warehousing. Decision Support 
Systems, 33, 143-161. http://dx.doi.org/10.1016/S0167-9236(01)00141-5

Nonaka, I. (1991). The knowledge creating company. Harvard Business Review, 96-104.

Nonaka, I. (1994). A dynamic theory of organizational knowledge creation. Organization Science, 5(1), 14-37. http://dx.doi.org/10.1287/orsc.5.1.14

Petro, J. (2012, March). Watson, I presume? Health and Management Technology, 8-10.

Rotman, D. (2013). How technology is destroying jobs. MIT Technology Review. Retrieved from http://www.technologyreview.com/featuredstory/515926/how-technology-is-destroying-jobs/

Schon, D. (1983). The reflective practitioner. New York: Basic Books.

Sharara, H. M. (1997). Toward resolving the predicaments of change: It's not "either/or" but rather "and". Comprehensive Evolution Management CEM. Retrieved from http://www.aradoportal.org.eg/Fuls_v5/Libraries/arado.aspx?fn=DLibApplySearch\&frameName=4.\&Scope $\mathrm{ID}=1.139 . \&$ criteria $1=2 . \&$ SearchText $1=$ Hussein + M. + Sharara

Sharara, H. M. (2014a). Managing innovation: Types of managers. Knowledge Management and Innovation. Ain Shams University.

Sharara, H. M. (2014b). Total mental fitness and 7.5 habits. Knowledge Management and Innovation. Ain Shams University, Cairo. $\quad$ Retrieved 12 December, 2014 from https://xa.yimg.com/kq/groups/.../10.+the+sven+and+half+habits.ppt

Taft, K. D. (2011, March). IBM's Watson: 'Jeopardy'win is just the beginning (pp. 22-23).

\section{Copyrights}

Copyright for this article is retained by the author(s), with first publication rights granted to the journal.

This is an open-access article distributed under the terms and conditions of the Creative Commons Attribution license (http://creativecommons.org/licenses/by/3.0/). 